\title{
Relationship between spontaneous non-propagating pressure activity in the oesophagus and acid gastro-oesophageal reflux in pathological and non- pathological refluxers
}

\author{
S KRUSE-ANDERSEN, L WALLIN, AND T MADSEN \\ From the Oesophagus Laboratory, Department of Thoracic and Cardiovascular Surgery T, Odense University \\ Hospital, Odense, Denmark
}

SUMmary To evaluate the oesophageal motor activity preceding episodes of reflux, 10 pathological and 10 non-pathological refluxers and 26 normal subjects were investigated. The pressure events in spontaneous short periods of pressure activity $(\leqslant 60 \mathrm{sec})$ and in long activity periods were registered. The last contraction before reflux was more frequently found non-propagating than the last contraction of pressure periods not followed by reflux $(p<0 \cdot 01)$. The interval from the last contraction to reflux was shortest, if the contraction terminated in, or confined to the upper part of the oesophagus $(\mathrm{p}<0 \cdot 001)$. Increased proportion of reflux episodes were preceded by an upper segmentary contraction $(\mathrm{p}<0 \cdot(05)$ and a short activity period $(\mathrm{p}<0 \cdot(02)$ in patients with pathological reflux in comparison with non-pathological refluxers. Spontaneously occurring sphincter relaxations might be triggered by preceding non-propagated contractile activity. The relative number of reflux episodes preceded by non-propagated pressure activity seems to be increased in patients with frequent episodes of acid reflux, compared with patients with infrequent episodes, or with normal subjects.

Episodes of acid gastro-oesophageal reflux have been shown to occur spontaneously in normal volunteers and in patients with duodenal ulcer. ${ }^{1-t}$ Duodenal ulcer patients have an increased reflux frequency and delayed acid clearance as compared with normal subjects. ${ }^{+}$In asymptomatic subjects as well as in patients with reflux disease spontaneous episodes of acid reflux occur predominantly by the mechanism of inappropriate gastro-oesophageal sphincter relaxations.': Episodes of acid reflux are frequently preceded within a short time by spontaneous oesophageal pressure activity."

The aim of the present investigation was to evaluate the quality of spontaneous oesophageal motor activity preceding acid gastro-oesophageal reflux episodes in a patient group with a known generally increased reflux tendency, but without oesophagitis.

Address for correspondence: $S$ Kruse-Andersen. Dept of Thoracic and (ardiovascular Surgery T. Odense University Hospital. Sdr Boulevard 29. DK-Odense ('. Denmark.

Reccived for publication 23 April 1987.

\section{Methods}

STUDY POPULATION

The material consisted of 20 duodenal ulcer patients, who within a month of the primary diagnosis underwent 12 hours of simultaneous monitoring of $\mathrm{pH}$ and pressure activity in the oesophagus. They were drawn from a population of duodenal ulcer patients referred for evaluation of acid gastro-oesophageal reflux. Patients with endoscopically proven oesophagitis were excluded. Two groups of 10 consecutive patients were selected to serve the purpose of the present study. Group one consisted of 10 patients with pathological acid gastro-oesophageal reflux ( $\mathrm{pH} \leq 4$ during more than $2.4 \%$ of the recording period), ${ }^{3}$ and group two comprised 10 patients without pathological acid gastro-oesophageal reflux. The two groups consisted of nine men and one woman, median age 58 and 37 years (range, 20-67 and 21-58 years), respectively. For comparison, the results from 26 normal volunteers, 18 with acid reflux, described in an earlier investigation, were used.' 
APPARATUS

A combined $\mathrm{pH}$ - and pressure probe (Radiometer GK $282 \mathrm{C}$ and two polyethylene catheters, Clay Adams PE 160) was introduced transnasally after local anaesthesia with Lidocain-spray ${ }^{\mathrm{R}}$. By means of manometric criteria, the probe was positioned and fixed with the electrode $5 \mathrm{~cm}$ proximally to the gastrooesophageal sphincter and the pressure catheters 8 and $23 \mathrm{~cm}$ proximally to the sphincter as described

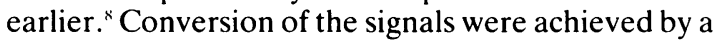
pH-meter (PHM 28 D) and microtip transducers (Millar PC-350) in connection with a continuous flush system (Intraflo R CFS 03) and transferred to an analogous tape recorder (Lyrec TR 47) as earlier described. ${ }^{\mathrm{x}}$ During playback with a time compression of 64 fold, the signals were printed out on a mingograph (Siemens Elema 803) with a paper speed of 10 $\mathrm{cm} / \mathrm{sec}$.

\section{EXPERIMENTAL CONDITIONS}

The monitoring was initiated one hour after a light meal and was then carried out between $8 \mathrm{pm}$ and $8 \mathrm{am}$. During this time the subjects were in the supine position and were not allowed to eat, drink, or smoke.

\section{DATA ANALYSIS}

A reflux episode was defined as a sudden fall $>2 \mathrm{pH}$ units to a $\mathrm{pH} \leq 4$. ${ }^{3}$ Pressure amplitudes were classified into propagating, simultaneous, reverse and upper and lower segmentary contractions. ${ }^{7 \times}$ When a distal pressure peak occurred after a proximal pressure peak within an interval of one to $5 \cdot 8 \mathrm{sec}$ a propagating peristaltic contraction was registered. A simultaneous contraction was defined as an interval between pressure peaks of one second or less. When a distal peak preceded a proximal peak within an interval of one to three seconds a reverse contraction was noted. Pressure peaks outside these limits were regarded as segmentary contractions. A relative pressure amplitude change of $8 \mathrm{~mm} \mathrm{Hg}$ and a rise time of $1 \mathrm{~mm} \mathrm{Hg} / \mathrm{sec}$ were required for a pressure peak to be registered."

The recorded pressure activity was divided into periods of contractile activity lasting $\leq 60 \mathrm{sec}$ (short activity periods) and periods with pressure activity greater than $60 \mathrm{sec}$. Contractions occurring within 30 sec of the preceding contraction were included in the same period. The end of a period of pressure activity was defined as a period of more than $30 \mathrm{sec}$ without any further pressure activity. ${ }^{7}$ Motor activity was defined as related to a reflux episode, if the time from the last contraction to the sudden fall in $\mathrm{pH}$ was less than $30 \mathrm{sec}^{79}$ An example of a short activity period related to an episode of reflux is shown in Figure 1. The number of the various types of peristaltic

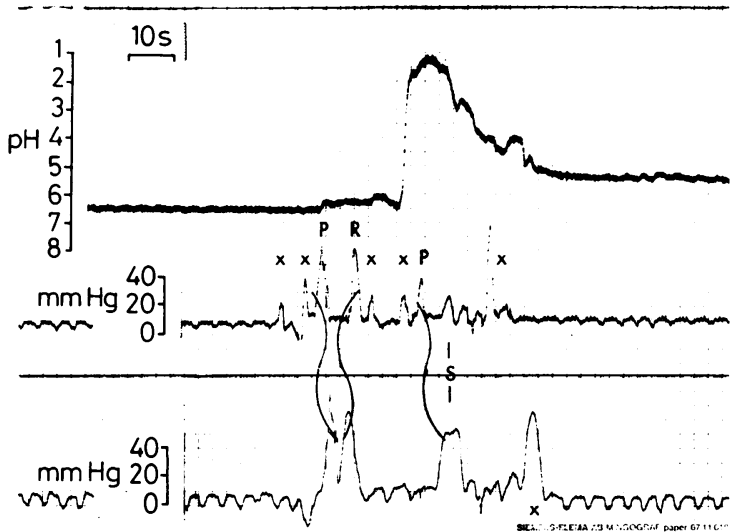

Fig. 1 Episode of reflux preceded by a short activity period terminating in an upper segmentary contraction. $(P)$ propagating pressure activity; $(S)$ simultaneous contraction; $(R)$ reverse pressure activity; $(x)$ segmentary contractions. Time related amplitudes are indicated.

contractions were counted irrespective of the length of the activity period or its relation to reflux. The type and velocity of the last contraction in short activity periods and of the last contractions before reflux were registered. The interval between a reflux episode and the pressure amplitude of a preceding contraction (if $<30 \mathrm{sec}$ ) was also measured.

STATISTICAL ANALYSIS

As a measure of quality the relative occurrence of the different types of contractile activity were used, assuming that this would at least fulfil the requirements for an ordinal scale. No tests requiring stronger assumptions were used and no assumptions were made regarding the distribution of the material. The Pratt's test was used in cases of two related samples. The Kruscal-Wallis and Friedmans nonparametric analysis of variances were used in cases of more than two independent or related samples, respectively. The Pratt's test and Friedmans test were used to test for interaction, which was considered present when the different groups made different contributions to the results when moving from one circumstance to another. For evaluation of correlation, the Spearmans rank correlation coefficient was calculated. In order to reduce the risk of committing errors of the first kind, a combination of values for the patient groups in cases of related samples and a combination of values for different circumstances in cases of independent samples were tested first, and exclusively in cases of significance, a multiple comparison procedure was carried out."'" p Values less than $0 \cdot 05$ were considered significant. 
Table Total number of reflux episodes and number of reflux episodes related io pressure activity, as well as number of short activity periods and total contractile activity during 12 hours of simultaneous registration of pH and pressure activity in 20 duodenal ulcer patients and 18 asymptomatic subjects

\begin{tabular}{|c|c|c|c|c|c|c|c|c|c|c|c|c|}
\hline & \multicolumn{3}{|c|}{ Reflux episodes (II) } & \multicolumn{3}{|c|}{$\begin{array}{l}\text { Reflux episodes after } \\
\text { pressure activity }(n)\end{array}$} & \multicolumn{3}{|c|}{$\begin{array}{l}\text { Short activity periods } \\
\text { unrelated to reflux }(n)\end{array}$} & \multicolumn{3}{|c|}{$\begin{array}{l}\text { Contractions during normal } \\
p H(t o t a l n)\end{array}$} \\
\hline & Total & Median & Range & Total & Median & Range & Total & Median & Range & Total & Median & Range \\
\hline $\begin{array}{l}\text { Asymptomatic subjects with } \\
\text { acid reflux }(n=18)\end{array}$ & 81 & 3 & $1-15$ & 67 & 3 & $0-15$ & 1.379 & 78 & $37-109$ & 10664 & 617 & $220-984$ \\
\hline $\begin{array}{l}\text { Duodenal ulcer without } \\
\text { pathological acid reflux } \\
(n=1())\end{array}$ & 53 & 5 & $0-18$ & 48 & 4 & $0-18$ & 974 & 91 & $76-143$ & 8463 & 757 & $459-1.394$ \\
\hline $\begin{array}{l}\text { Duodenal ulcer with } \\
\text { pathological acid reflux } \\
(n=10)\end{array}$ & 2015 & 19 & $11-38$ & 184 & 16 & $8-33$ & 6.52 & 62 & $27-103$ & 5723 & 454 & $309-1186$ \\
\hline
\end{tabular}

\section{Results}

The number of reflux episodes and the pressure activity in the different subjects groups are shown in the Table. Two hundred and ninety nine episodes of acid reflux were preceded within 30 sec by pressure activity. Fifty nine per cent were preceded by short periods of pressure activity, $27 \%$ by long activity periods, and $15 \%$ by a single contraction.

The relative number of the different types of contractions in activity periods before reflux were not significantly different from the number in activity periods not followed by a fall in $\mathrm{pH}$. The last contraction of pressure periods before reflux

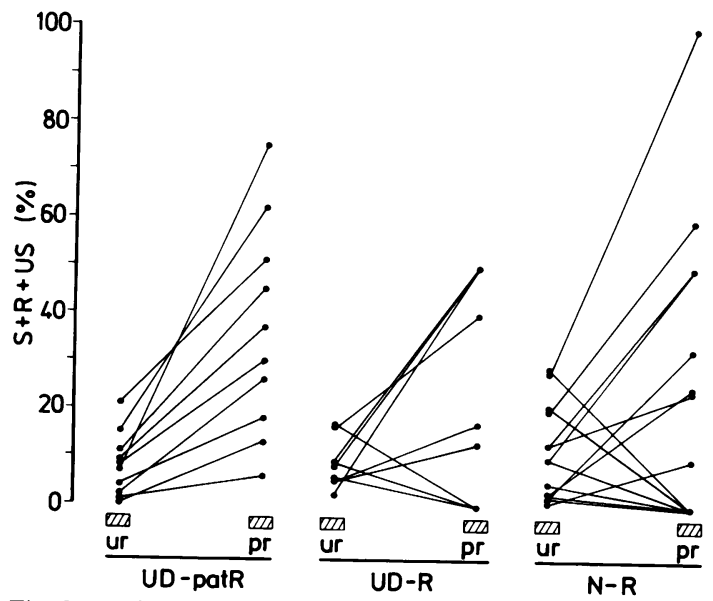

Fig. 2 Relative frequency of simultaneous $(S)$, reverse $(R)$, and upper segmentary (US) pressure waves. (ur) last contraction of short activity periods unrelated to reflux; (pr) last contraction prior to all reflux episodes preceded by pressure activity; (UD-patR) duodenal ulcer patients with pathological acid reflux; (UD)-R) duodenal ulcer patients presenting with reflux within the normal range; $(N-R)$ asymptomatic subjects with at least one episode of reflux preceded by pressure activity. episodes, however, consisted more frequently of a simultaneous, reverse or upper segmentary contraction than the last contraction of short activity periods not followed by reflux $(\mathrm{p}<0 \cdot 01)($ Fig. 2$)$, and this was also true when only short activity periods prior to reflux were considered (Fig. 3). Patients with pathological reflux made a greater contribution to this difference than the other groups $(p<0 \cdot 05)$. The interval from the last contraction to a fall in $\mathrm{pH}$ was shorter, if the contraction was terminated in or confined to the upper part of the oesophagus (simultaneous, reverse or upper segmentary) compared with propagating or lower segmentary contractions $(\mathrm{p}<0 \cdot 001)$ (Fig. 4). This difference was most marked in subjects without pathological reflux $(p<0 \cdot 05)$. In cases of propagating pressure activity before reflux no correlation could be shown between the peristaltic velocity and time to reflux.

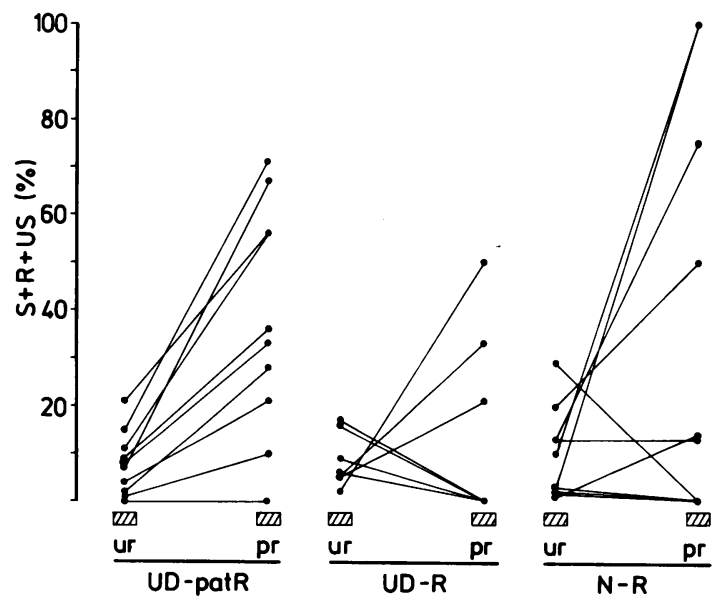

Fig. 3 Relative frequency of simultaneous $(S)$, reverse $(R)$, and upper segmentary (US) pressure waves. (ur) last contraction of short activity periods unrelated to reflux; (pr) last contraction of short activity periods prior to reflux. Remaining abbreviations as in Fig. 2. 
The contraction frequency was greater during short activity periods preceding reflux than during long activity periods before reflux or any type of period unrelated to reflux $(\mathrm{p}<0 \cdot(0) 1)$ irrespective of subject group. The last contraction of a short activity period without reflux was more likely to be progressive than the pressure activity within the rest of the period $(\mathrm{p}<0 \cdot(0) 1)$, regardless of subject group (Fig. 5).

Relatively more reflux episodes followed pressure periods terminated by an upper segmentary contraction in patients with pathological reflux as compared with the two groups without pathological reflux $(p<0 \cdot(05)$. In addition, a relatively higher number of reflux episodes were preceded by a short activity period in patients with pathological reflux as compared with non-pathological refluxers $(p<0 \cdot(02)$ (Fig. 6).

\section{Discussion}

In normal subjects a decreased resting pressure in the gastro-oesophageal sphincter has been shown to play a minimal role in the production of gastrooesophageal reflux episodes. Dent et al' found this factor responsible in less than $2 \%$ of reflux episodes in healthy subjects. In patients with reflux induced oesophagitis Dodds et al found that only one third of the recorded reflux episodes could be attributed to a low resting sphincter pressure or a low sphincter pressure in combination with increased intragastric

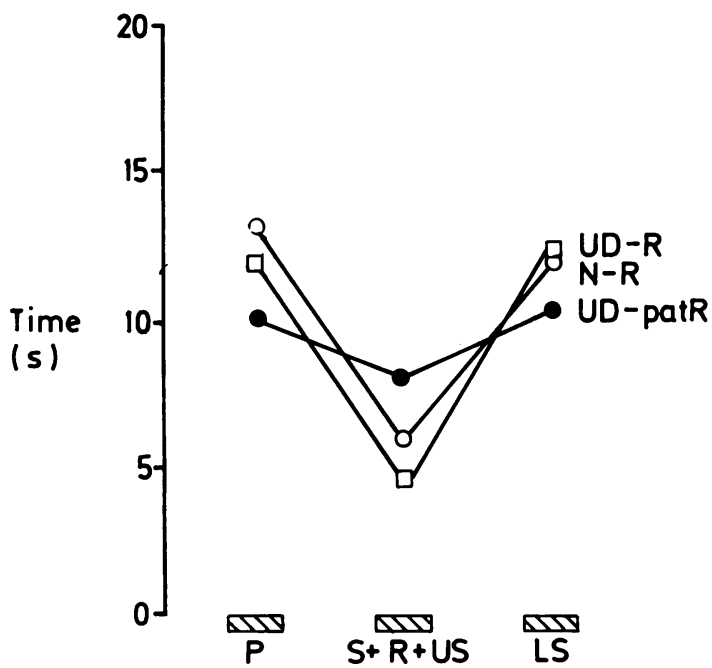

Fig. 4 Intervals from pressure activity to reflux. $(P)$ propagating pressure waves; $(S)$ simultaneous contractions; (R) reverse pressure waves; (US) upper segmentary contractions; (LS) lower segmentary contractions. Unbroken lines are related median values for the three subject groups. Remaining abbreviations as in Fig. 2. pressure. Most of the spontaneously occurring reflux episodes recorded in normal subjects and patients with gastro-oesophageal reflux disease occurred during inappropriated relaxation of the gastrooesophageal sphincter - that is, sphincter relaxation not triggered by the passage of a bolus. ${ }^{15}$ : Sphincter relaxation and reflux are frequently preceded by peristaltic activity. " but a proportion of the contractions preceding reflux are not propagating peristalsis elicited by swallowing. " ": These observattions suggest that reflux may result from a sphincter relaxation triggered by a non-propagating peristaltic contraction or insufficiently bolus transporting propagating peristalsis. Acid reflux during the night is probably the most important factor in the development of reflux changes in the oesophagus." Acid clearance both in normal subjects and in patients with reflux disease is prolonged during the night. "' Reflux episodes and oesophageal contractile activity during the night have been shown to occur during transient arousal from sleep.' contractions during the night occurring predominantly in brief bursts. ${ }^{7}$ An increased occurrence of non-propagating contractions in these clusters of activity has been observed.' In dog experiments triggering of lower oesophageal sphincter relaxations have been achieved by stimulation of the striated upper portion of the oeosophagus. ${ }^{15}$ We therefore evaluated the relationship between reflux episodes and the activity pattern in

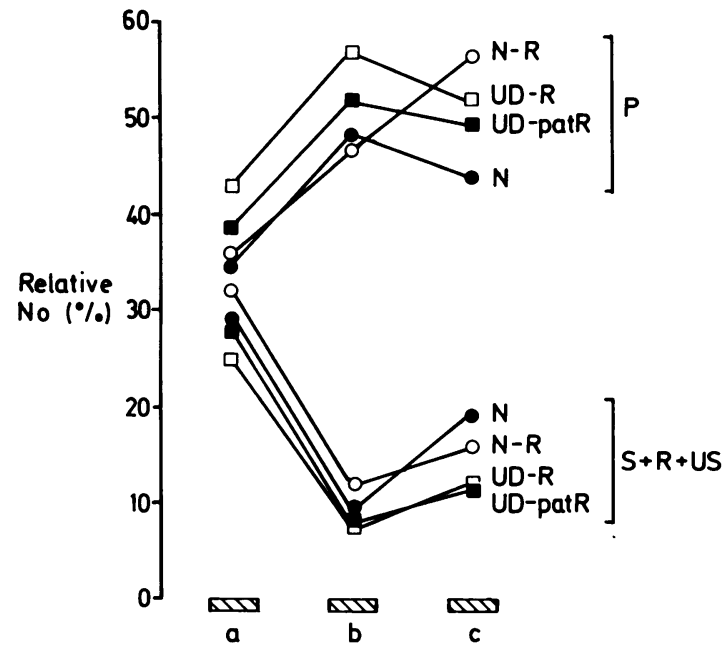

Fig. 5 Pattern of pressure activity unrelated to reflux episodes. Relative frequency of propagating $(P)$ and nonpropagating $(S+R+U S)$ pressure activity, when considering the initial part (a) and the last contraction (b) in short activity periods as well as the remaining pressure activity (c). Unbroken lines are related median values for the four subject groups. $(N)$ asvmptomatic subjects without reflux during the monitoring period. Remaining abbreviations as in Fig. ?. 


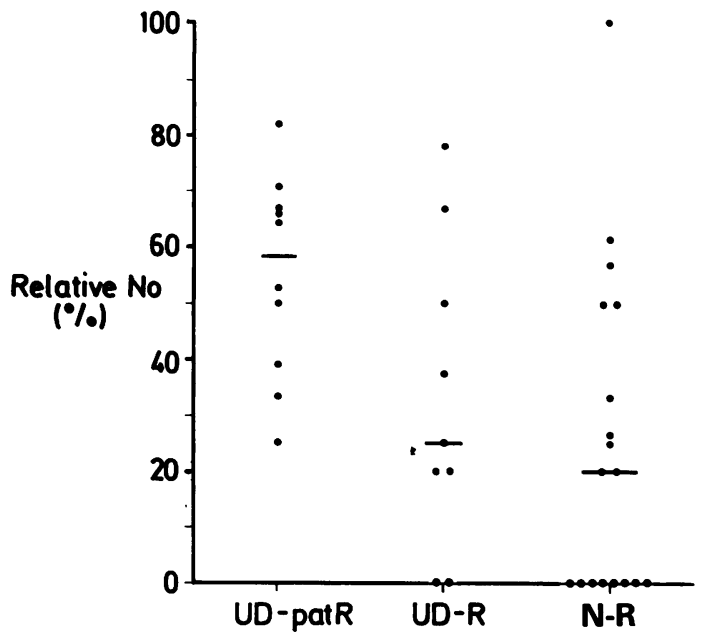

Fig. 6 Relative number of reflux episodes preceded by short activity periods in patients with (UD-patR) and without $(U D-R)$ pathological reflux and in normal subjects with acid reflux $(N-R)$. Horizontal bars denote median values.

the preceding periods of pressure activity. By positioning one catheter in the upper portion of the oesophagus $23 \mathrm{~cm}$ proximally to the gastrooesophageal sphincter contractions elicited by a swallow, but not conducted to the lower oesophagus, could be recorded.

The concept of a relationship between nonpropagating pressure activity and sphincter inhibition is supported by the finding in this study of a frequent change in the termination of short periods of activity, when occurring before reflux compared with short activity periods unrelated to reflux. This increase in non-propagated activity could also be observed when the last contraction before reflux occurred other than in short periods of pressure activity. An increased contraction frequency during short activity periods before reflux suggests that a cluster of ineffective contractions terminating in a non-propagated contraction wave might cause sphincter relaxation. Short activity periods showed an increased content of non-propagated waves but when they were not followed by an episode of reflux, such bursts were more likely to terminate in a propagated contraction. Under these circumstances relaxation of the sphincter would be followed by bolus clearing peristalsis and closure of the sphincter.

In our study, short periods of activity were more frequent before reflux than long activity periods or single contractions. Reflux during long periods of pressure activity were not preceded by an increased frequency of pressure waves. In these cases sphincter inhibition may have been by another mechanism, especially when the last preceding contractions were propulsive. On the other hand reflux preceded by propagating peristalsis might in some cases occur by the above mentioned mechanism, as the bolus transporting efficiency of propagating peristalsis was not measured. This is in accordance with Dent et al who found that when reflux occurred after propagating peristalsis the sphincter shortly recovered its tone before an inappropriate relaxation resulted in reflux. In contrast, when reflux occurred in relation to a failed peristaltic sequence, it followed shortly after swallowing. In the present material about one third of the recorded reflux episodes were preceded by a propagating peristaltic wave, which is in accordance with the findings by others. ${ }^{\prime \prime}$

In patients with pathological reflux, a greater proportion of reflux episodes were preceded by an upper segmentary contraction and a short activity period, as compared with subjects without pathological reflux. In addition, a change in the quality of the last contraction could be measured more frequently in this patient group when comparing periods of pressure activity related to and unrelated to reflux. These findings are consistent with previous observations in patients with reflux oesophagitis. ${ }^{5}$ '2

It is concluded that in subjects with or without pathological acid gastro-oesophageal reflux, a part of the spontaneously occurring sphincter relaxations producing episodes of acid gastro-oesophageal reflux might be triggered by preceding non-propagating pressure activity. The relative number of reflux episodes preceded by non-propagating pressure activity seems to be increased in patients with frequent episodes of acid reflux as compared with patients with infrequent reflux episodes or normal subjects.

\section{References}

1 Dent J, Dodds WJ, Friedman RH, et al. Mechanism of gastroesophageal reflux in recumbent asymptomatic human subjects. J Clin Invest 1980; 65: 256-67.

2 Boesby S. Gastro-oesophageal acid reflux and sphincter pressure in normal human subjects. Scand J Gastroenterol 1975; 10: 731-6.

3 Wallin L, Madsen T. 12-hours simultaneous registration of acid reflux and peristaltic activity in the oesophagus. A study in normal subjects. Scand J Gastroenterol 1979; 14: $561-6$.

4 Wallin L. Gastro-oesophageal function in duodenal ulcer patients. Scand J Gastroenterol 1980; 15: 145-50.

5 Dodds WJ, Dent J, Hogan WJ, et al. Mechanisms of gastroesophageal reflux in patients with reflux esophagitis. $N$ Engl J Med 1982; 307: 1547-52.

6 Corazziari E, Bontempo I, Anzini F, Torsoli A. Motor activity of the distal oesophagus and gastro-oesophageal reflux. Gut 1984; 25: 7-13.

7 Kruse-Andersen S, Wallin L, Madsen T. The quality of oesophageal peristalsis preceding acid gastro- 
oesophageal reflux episodes. A study in normal subjects. Scand J Gastroenterol 1986; 21: 711-7.

8 Wallin L. Madsen T. Boesby S, Sørensen O. Gastrooesophageal acid reflux and oesophageal peristalsis. Method for 12-hour simultaneous recording of $\mathrm{pH}$ and peristaltic activity in the oesophagus. Scand J Gastroenterol 1979; 14: 481-7.

9 Madsen T. Wallin L, Boesby S, Larsen VH. Oesophageal peristalsis in normal subjects. Influence of $\mathrm{pH}$ and volume during imitated gastro-oesophageal reflux. Scand J Gastroenterol 1983; 18: 513-8.

10 Bradley JV. Distribution-free statistical tests. Englewood Cliffs: Prentice-Hall, 1968.

11 Conover WJ. Practical non parametric statistics. Wiley: New York, 1971.
12 Baldi F. Ferrarini F. Balestra R. et al. Oesophageal motor events at the occurrence of acid reflux and during endogenous acid exposure in healthy subjects and in patients with oesophagitis. Gut 1985; 26: 33641.

13 Orr WC. Robinson MG, Johnson LF. Acid clearance during sleep in the pathogenesis of reflux esophagitis. Dig Dis Sci 1981: 26: 423-7.

14 Orr WC. Johnson LF, Robinson MG. Effect of sleep on swallowing, esophageal peristalsis, and acid clearance. Gastroenterology 1984; 86: 814-9.

15 Price LM, El-Sharkawy TY, Mui HY, Diamant NE. Effect of bilateral cervical vagotomy on balloon-induced lower esophageal sphincter relaxation in the dog. Gastroenterology 1979; 77: 324-9. 